\section{Duration of remitting psychoses with acute onset}

\author{
Implications for ICD-I0
}

RAMIN MOJTABAI, VIJOY K. VARMA and EZRA SUSSER

\begin{abstract}
Background The acute and transient psychotic disorders (ATPD) in ICD-10 advanced the nosology of remitting psychoses with acute onset. But the proposed criteria for ATPD - especially in regard to duration - are tentative and need to be validated.
\end{abstract}

\begin{abstract}
Aims To evaluate: (a) the duration of remitting psychoses with acute onset; (b) the applicability of the ATPD criteria for these cases; and (c) differences in duration and ATPD diagnoses across sociocultural settings.
\end{abstract}

Method Data from the World Health study were used.

\section{Results The 98 cases of remitting psychoses with acute onset had a modal duration of 2-4 months, with $43 \%$ falling in this range. Mainly because of this, few met the ATPD criteria. Duration and diagnostic findings were similar across settings.}

\section{Conclusions ATPD criteria need refinement, especially in regard to duration. Further studies aimed at early detection and assessment of onset and duration of these disorders are needed.}

\section{Declaration of interest Partial} support detailed in Acknowledgements. No conflict of interest. Organization Determinants of Outcome
The tenth edition of the International Classification of Diseases (ICD-10) (World Health Organization, 1992) advanced the study of remitting psychoses with acute onset by proposing the tentative diagnoses of acute and transient psychotic disorders (ATPD). These include four specific and two non-specific disorders (Table 1). Despite some early attempts to validate these diagnoses (Jørgensen et al, 1997; Malhotra et al, 1998), their boundaries remain elusive. Results of studies by our group suggested that remitting psychoses with acute onset typically last longer than the maximum 1-3 months allowed for ATPD, and that the duration might be different between developing and industrialised settings (Susser et al, 1995a,b). Following this, the present study examines the duration and the use of ATPD criteria across settings in a large international sample in which we previously delineated remitting psychoses with acute onset from schizophrenia through their distinct epidemiological characteristics (Susser \& Wanderling, 1994).

\section{METHOD}

The methods of the Determinants of Outcome of Severe Mental Disorders (DOSMeD) study are described elsewhere (Jablensky et al, 1992). Briefly, in defined catchment areas in 13 sites, investigators were to include all first-contact patients presenting with a psychotic disorder to a helping agency over a 2-year period. Patients ranged in age from 15 to 54 years.

The 13 sites were classified by the original investigators of the DOSMeD into five developing and eight industrialised country sites (Jablensky et al, 1992). Developing country sites included Agra, India; Cali, Colombia; urban Chandigarh, India; rural Chandigarh, India; and Ibadan, Nigeria. The industrialised country sites included Aarhus, Denmark; Dublin, Ireland; Honolulu, Hawaii; Moscow, Russia; Nagasaki, Japan; Nottingham, England; Prague, the Czech Republic; and Rochester, NY, USA. In 8 of the 13 sites, the coverage of the catchment area was more comprehensive, and the sample of patients from these sites was considered an incidence cohort (Jablensky et al, 1992). These eight sites included two developing country sites (rural Chandigarh and urban Chandigarh) and six industrialised country sites (Aarhus, Dublin, Honolulu, Moscow, Nagasaki, and Nottingham). Note that both developing country incidence sites are in the same region of India; nevertheless, they represent different sociocultural contexts. The rural catchment area in Chandigarh is a green fertile region in Ambala District (Harayan state), which, although more prosperous than many rural Indian locales, has a $56 \%$ illiteracy rate. Urban Chandigarh, on the other hand, is one of the more modern cities in India, with a population of about 348000 in 1980 (Day et al, 1987; Jablensky et al, 1992; Collins et al, 1999).

The sample for this study, as in our previous study (Susser \& Wanderling, 1994), was drawn from a subset of 794 subjects in the DOSMeD cohort (444 in the eight incidence sites) who met the following inclusion criteria: full intake assessment,
Table I Sub-classification of acute and transient psychotic disorders (F23) in ICD-10

\begin{tabular}{lc}
\hline Categories & Duration \\
\hline Specific categories & \\
Acute polymorphic psychotic disorder without symptoms of schizophrenia (F23.0) & $<3$ months \\
Acute polymorphic psychotic disorder with symptoms of schizophrenia (F23.I) & $<$ I month \\
Acute schizophrenia-like psychotic disorder (F23.2) & $<$ I month \\
Other acute predominantly delusional psychotic disorders (F23.3) & $<3$ months \\
Non-specific categories & Not specified \\
Other acute and transient psychotic disorders (F23.8) & Not specified \\
Acute and transient psychotic disorder, unspecified (F23.9)
\end{tabular}


completion of a 2-year follow-up, an ICD-9 diagnosis of schizophrenia at study entry, and no clear-cut affective (or other nonpsychotic) syndrome during the 2-year follow-up period.

\section{Research instruments}

At entry to the study, and at the 2-year follow-up, a research psychiatrist administered the ninth edition of the Present State Examination (PSE; Wing et al, 1974) and the Diagnostic and Prognostic Schedule (DPS; Jablensky et al, 1992), and on the basis of these ratings made an ICD-9 diagnosis. In addition, the Psychiatric and Personal History Schedule (PPHS; Jablensky et al, 1992), usually completed with a key informant, was administered by a mental health professional, often a psychiatric social worker. The interrater reliability for ratings used in the present analyses have been previously reported (Jablensky et al, 1992) and were all well within the acceptable range.

\section{Selection of subjects}

For the examination of duration, subjects were selected who had experienced psychosis with acute onset, complete remission and no relapses within the 2-year followup period. These cases are henceforth called remitting psychoses with acute onset. Cases with multiple episodes within the 2-year follow-up period were excluded, because the duration of each individual episode was not recorded in the DOSMeD study.

Onset, course and duration were assessed using specific ratings on the PPHS administered at baseline and at 2-year follow-up. Acute onset (termed 'sudden onset' in the PPHS) was operationalised as "a florid psychotic state developed within days (up to a week), without being preceded by any prodromal signs or symptoms". Complete remission and no relapse was operationalised as "a single psychotic episode followed by a complete remission", rated at the 2-year follow-up.

Of 794 patients in all 13 sites of the DOSMeD study who had a full initial evaluation, a 2-year follow-up assessment, an ICD-9 schizophrenia diagnosis at study entry, and no clear-cut affective syndrome during the follow-up period, $98(12 \%)$ also met the criteria for remitting psychoses with acute onset as described above. The distribution of cases of remitting psychoses with acute onset according to gender and site is presented in Table 2 . Whereas $21 \%$
(78 out of 376 ) of patients in the developing country settings were categorised as cases of remitting psychoses with acute onset, only $5 \%$ (20 out of 418 ) in the industrialised settings could be so categorised.

\section{Examination of duration}

Duration of illness was computed as the sum of two ratings made on the PPHS: (a) the duration of illness before intake, rated in months; and (b) the duration of illness during the 2-year follow-up period, rated in weeks (converted to months). We examined the duration of illness in all selected subjects by plotting the duration of illness in 2-month intervals on histograms. First, we examined the distribution in all sites. Next, we examined the distribution of duration in the developing $v$. industrialised sites separately. Finally, to assess the possibility that the incidence sites might have captured cases with shorter duration which were missed by non-incidence sites, we examined the duration in the eight incidence sites where the ascertainment of cases was more complete.

\section{ICD-I0 diagnosis}

As part of a 15-year follow-up of the original DOSMeD cohort, psychiatrists at each centre re-diagnosed the patients using ICD-10 criteria (Sartorius et al, 1996). For this, they used all available information on each patient. We requested ICD-10 diagnoses from incidence sites for patients with remitting psychoses with acute onset whose illness had lasted 6 months or less. ICD-10 diagnoses for five sites - rural Chandigarh, urban Chandigarh, Nottingham, Dublin and Moscow - are reported here. In two of the incidence sites (Nagasaki and Honolulu), no cases met the inclusion criteria described earlier. The Aarhus site did not take part in the 15-year follow-up study, therefore no ICD-10 diagnoses were available for this centre.

\section{RESULTS}

\section{Duration}

Figure 1(a) shows the distribution of duration for the 98 cases of remitting psychoses with acute onset in all sites. These cases had a modal duration of $2-4$ months with $43 \%$ $(n=42)$ of the cases falling within this range. Thirty $(31 \%)$ cases had a duration of 3 months or less, and only one case $(1 \%)$ had a duration of 1 month or less.
Table 2 Distribution of remitting psychoses with acute onset according to setting and gender in the Determinants of Outcome of Severe Mental Disorders study

\begin{tabular}{|c|c|c|c|}
\hline Centre & $\begin{array}{l}\text { No. of } \\
\text { females }\end{array}$ & $\begin{array}{l}\text { No. of } \\
\text { males }\end{array}$ & $\begin{array}{c}\text { Total } \\
\text { no. }\end{array}$ \\
\hline \multicolumn{4}{|l|}{ Developing country } \\
\hline Agra & 10 & 16 & 26 \\
\hline Cali & 3 & 5 & 8 \\
\hline Ibadan & 9 & 8 & 17 \\
\hline Chandigarh, urban' & II & 4 & 15 \\
\hline Chandigarh, rural' & 5 & 7 & 12 \\
\hline Total & 38 & 40 & 78 \\
\hline \multicolumn{4}{|l|}{ Industrialised country } \\
\hline Aarhus' & I & 0 & I \\
\hline Moscow' & 3 & I & 4 \\
\hline Honolulu' & 0 & 0 & 0 \\
\hline Rochester & 0 & 0 & 0 \\
\hline Prague & 6 & I & 7 \\
\hline Nagasaki' & 0 & I & I \\
\hline Nottingham' & 4 & $\mathrm{I}$ & 5 \\
\hline Dublin' & I & $\mathrm{I}$ & 2 \\
\hline Total & 15 & 5 & 20 \\
\hline
\end{tabular}

I. Incidence sites.

Figures 1(b) and 1(c) show the distribution of duration for the 78 patients from the developing country settings and 20 from the industrialised country settings separately. The pattern was similar across the two settings.

Figure 1(d) shows the distribution of duration for the 40 cases from the eight incidence sites. The pattern was similar to the distribution in all 13 sites.

\section{ICD-10 diagnoses}

As noted earlier, we requested ICD-10 diagnoses for patients from incidence sites with remitting psychoses with acute onset, whose illness lasted 6 months or less. Thirty patients in both settings met these criteria. Of these, $10(33 \%)$ were from the industrialised country settings and $20(67 \%)$ from the developing country settings. Eleven $(37 \%)$ patients were male and 19 $(63 \%)$ female. ICD-10 diagnoses were available for $27(90 \%)$ of these patients. In the Moscow site, only patients recruited in the first year of the 2 years of the study recruitment were in the 15-year follow-up; as a result two patients from this site did not receive ICD-10 diagnoses. One patient was from the Aarhus site which, as noted 
(a)

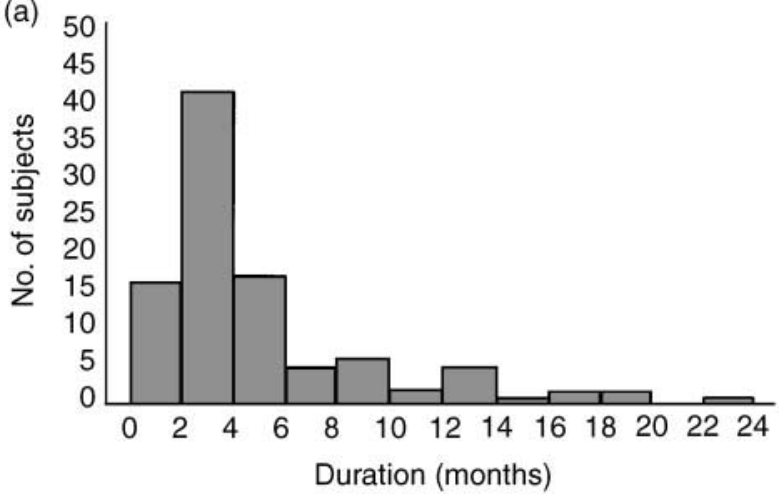

(c)

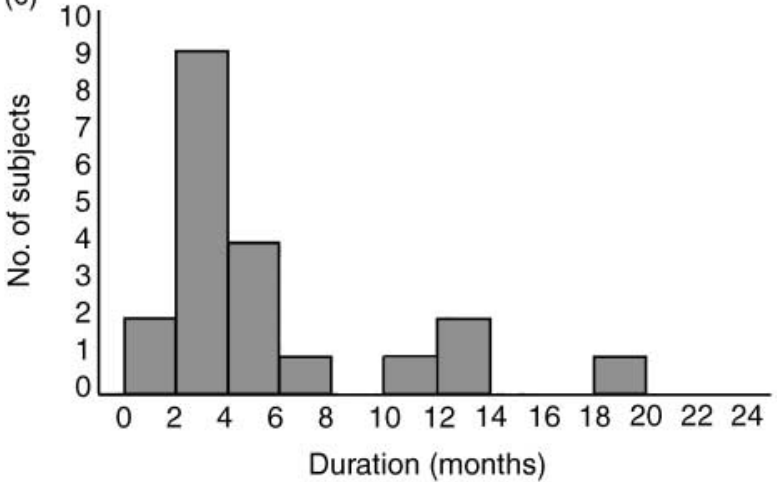

(b)

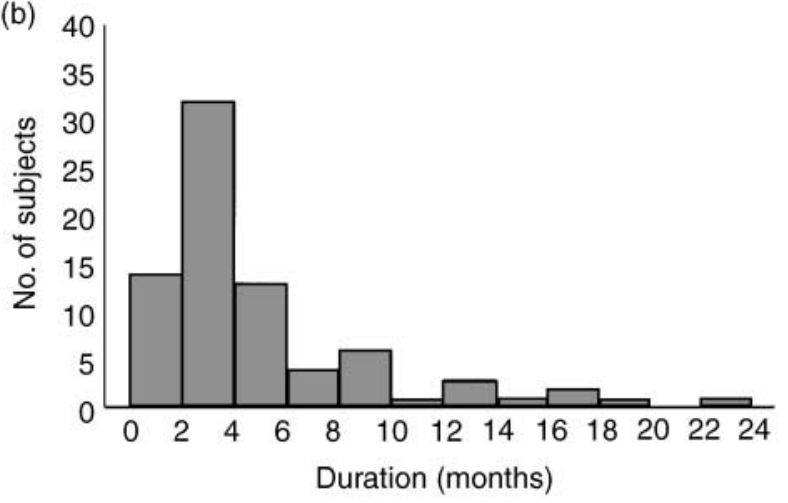

(d)

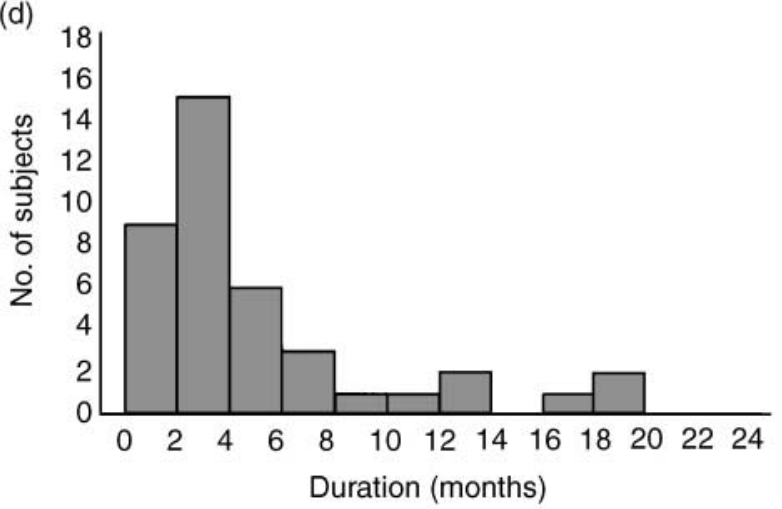

Fig. I The distribution of duration of remitting psychoses with acute onset in the Determinants of Outcome of Severe Mental Disorders study for: (a) the 98 cases in all of the 13 sites; (b) the 78 cases in developing country settings; (c) the 20 cases in industrialised country settings; and (d) the 40 cases in the eight incidence sites.

earlier, was not included in the follow-up study.

Diagnoses for the 27 cases were as follows: 16 (59\%) schizophrenia (F20.0, F20.1, F20.3, F20.5, F20.9); four (15\%) other acute and transient psychotic disorder (F23.8); two (7\%) acute schizophrenia-like psychotic disorder (F23.2); one (4\%) other non-organic psychotic disorder (F28); one (4\%) recurrent depressive disorder with psychotic symptoms (F33.3); one (4\%) mania with psychotic symptoms (F30.2); one $(4 \%)$ alcoholic hallucinosis (F10.5); and one $(4 \%)$ mental and behavioural disorder due to use of other stimulants (F15.5).

\section{DISCUSSION}

There were three major findings in this study. The first was that remitting psychoses with acute onset had a modal duration of 2-4 months - longer than the maximum 1-3 months (depending on the specific diagnosis) allowed for the ICD-10 diagnoses of ATPD. Cases from the incidence sites, where ascertainment extended to first contacts with general practitioners and faith healers, had a distribution similar to that of cases from the rest of the $\mathrm{DOSMeD}$ sites. Therefore, this finding is unlikely to be explained by a failure to capture the cases with shorter duration.

The second finding was that the distribution of duration was similar between sites classified as 'developing' and 'industrialised' by the original investigators (Jablensky et al, 1992). Earlier findings from different samples had suggested the possibility that remitting psychoses with acute onset might have a longer duration in the developing settings than in the industrialised settings (Susser et al, 1995a,b). However, these earlier suggestive findings were not corroborated by the present study, which directly compared the duration between the two settings.

The third finding was that only two cases $(7 \%)$ of the 27 with an ICD-10 diagnosis met the criteria for one of the specific ATPD, and four (14\%) met the criteria for a non-specific ATPD. The most common diagnosis for the other cases was schizophrenia $(n=16,59 \%)$. This was largely because the duration of these cases exceeded the maximum allowable duration for ATPD (1-3 months, depending on the specific diagnosis). Again, this pattern was consistent in both developing and industrialised settings. In fact, no cases met the ATPD criteria in the industrialised settings.

\section{Implications for ICD-I0 ATPD}

The introduction of ATPD in ICD-10 was an advance over other attempts at classifying remitting psychoses. The ICD-10 formulation was influenced by the classic descriptions by continental psychiatrists of such psychoses under different names such as 'cycloid psychoses' (Leonhard, 1961) and 'bouffées délirantes' (Pichot, 1986). The growing empirical evidence for the distinctive epidemiological characteristics (Susser \& Wanderling, 1994), long-term course (Susser et al, 1998), biological correlates (Collins et al, 1999), and, as reported here, distinctive distribution of duration, all support the ICD-10 concept of ATPD as a nosological entity distinct from other psychotic disorders. 
However, the operational criteria for ATPD in ICD-10 need refinement, so as to better capture the cases of remitting psychoses with acute onset for which they were designed. In this study, none of the cases of remitting psychoses with acute onset in the industrialised country settings, and only two cases in the developing country settings, were allocated to one of the specific ATPD categories.

We have elsewhere proposed specific revisions for ICD-10 criteria (Susser et al, 1998). One revision, supported by the results of the present study and our previous work (Susser et al, 1995b), is to extend the duration criterion to 6 months. Another revision proposed is to forego the current sub-classification within the ATPD pending further research. Currently ICD10 includes four specific and two nonspecific disorders under the rubric of the ATPD (Table 1). However, as noted in the text of the ICD-10, there is little empirical evidence for such sub-classification. The small number of cases which met the criteria of ATPD in the present study did not allow us to examine the validity of this sub-classification. Indeed, as these conditions are rare in industrialised countries, where most diagnostic research is conducted, the number of patients with psychosis who would be needed to collect enough cases of each specific ATPD for validation studies would be prohibitively large. Partly for this reason, we and other authors (Susser et al, 1995b; Jørgensen $e t$ al, 1997) have questioned the usefulness of dividing ATPD into distinct disorders.

A fundamental difference between the ICD-10 system and the DSM-IV (American Psychiatric Association, 1994) system in regard to classification of brief psychoses is the inclusion of mode of onset as a criterion. ICD-10 ATPD are defined by acute onset, whereas DSM-IV schizophreniform disorder and brief psychotic disorder are not. A further analysis of the DOSMeD data showed that remitting psychoses with acute onset have an epidemiological distribution quite distinct from that of the remitting psychoses with non-acute onset (unpublished data available from the first author upon request). Remitting psychoses with acute onset were approximately twice as common in women and 10 times as common in the developing country settings; this was not found, however, for remitting psychoses with non-acute onset.

\section{Implications for other ICD-I0 diagnoses}

Duration criteria are used to distinguish two of the ATPD diagnoses (F23.1 and F23.2; see Table 1) from schizophrenia (F20), and two other ATPD diagnoses (F23.0 and F23.3; see Table 1) from persistent delusional disorders (F22) and other non-organic psychotic disorders (F28). Revision of the ATPD duration criteria, therefore, may have repercussions on the diagnostic criteria of the other disorders. For example, if the maximum duration of ATPD is extended to 6 months, the minimum duration criterion for schizophrenia may also need to be extended to 6 months. The implications of changing the duration criteria for ATPD on the boundaries with other disorders need to be empirically assessed in future studies.

\section{Directions for future research}

As the findings from this study suggest, there is a need for additional research on remitting psychoses with acute onset. Future research will have to be on a large scale and involve a number of centres, because of the relative rarity of these disorders and because of the potential importance of the sociocultural setting. Special attention will have to be given to defining the concept of acute onset, and it seems likely that this will involve using many different sources of information, in spite of the inevitable practical difficulties.

Future research also needs to examine the symptoms of these psychoses in more depth. Classic descriptions of remitting psychoses with acute onset, such as cycloid psychoses (Leonhard, 1961) and boufées délirantes (Pichot, 1986), gave prominence to the use of distinctive symptoms in differentiating these disorders from other psychotic disorders. Some of these features, such as polymorphic symptoms and perplexity, are mentioned in the description of ATPD in ICD-10, and if future research is successful in identifying a set of reliable and specific symptoms for these disorders, such features may be given more prominence in the future ICD editions. However, very few modern empirical studies have attempted to identify symptoms that are specific to remitting psychoses with acute onset and can distinguish these cases from cases of other psychotic disorders, and, in our own research, we have had little success in identifying such symptoms.

\section{Limitations}

The results of this study should be considered in the context of its limitations. First, the number of developing country incidence sites was limited to two, and the majority of the cases of remitting psychoses with acute onset were identified in these two sites. It is reassuring, however, that the examination of duration in the developing and in the industrialised settings in the larger sample, which included all sites, revealed remarkably similar results across the two settings as well.

Second, the criteria for ICD-10 ATPD were not developed at the time of the $\mathrm{DOSMeD}$ study and these disorders were not the focus of this study; therefore, no questions about the specific criteria of these disorders were included. This is particularly the case in regard to the rating of mode of onset. Acute onset in ICD-10 is operationalised as onset within 2 weeks. The closest rating to this in the DOSMeD study was the rating of onset within 1 week. The findings from the present study need to be replicated in more focused and detailed studies using specific ICD-10 criteria.

Third, ratings of mode of onset and duration of illness were often based on information obtained from a key informant who was available for the interview, and these data could not always be verified by other means (such as using multiple sources of information). However, these ratings were made using standardised instruments with established reliability within and across sites (Jablensky et al, 1992).

Fourth, an ICD-9 diagnosis of schizophrenia was among the inclusion criteria for the present study. This excluded cases of ICD-9 reactive psychosis (codes 298.3, 298.4 and 298.8), even if these cases met the other criteria for remitting psychoses with acute onset. In the incidence sites of the $\mathrm{DOSMeD}$, however, only one case out of the 43 with a diagnosis of reactive psychosis met the onset and duration criteria for remitting psychoses with acute onset as defined in this study. Inclusion of this case in the analyses did not change the results.

Fifth, the duration of illness before intake into the study was rated in months. This rating was less fine-grained than the rating of duration after intake, which was made in weeks. However, the median duration of illness before intake in both settings was only 1 month, and the median duration 
after intake was 9 weeks. More precise assessment of duration before intake, therefore, could not have changed the results of the study.

Sixth, as described earlier, for methodological reasons, cases with more than one episode during the 2-year follow-up were not included in the study. Both the classic descriptions of remitting psychoses with acute onset (Leonhard, 1961; Pichot, 1986) and the modern clinical studies (e.g. Guinness, 1992) have noted recurrence of episodes in a subgroup of these patients. The results of the present study, therefore, need to be replicated in studies which include patients with multiple episodes.

\section{ACKNOWLEDGEMENTS}

We would like to acknowledge the contribution of the 12 World Health Organization Field Research Centers that carried out the Determinants of Outcome Study. The chief collaborating investigators were E. Strömgren, Aarhus; K. C. Dube, Agra; C. Leon, Cali; N. N. Wig and V. Varma, Chandigarh; D. Walsh, Dublin; A. Marsella and M. Katz, Honolulu; M. Olatawura, Ibadan; R. A. Nadzharov and N. N. Zharikov, Moscow; R. Takahashi and Y. Nakane, Nagasaki; J. Cooper, Nottingham; L. Hanzlicek and C. Skoda, Prague; and L.C.Wynne and T. Gift, Rochester. At the World Health Organization Headquarters, the study was coordinated by N. Sartorius, principal investigator, and A. Jablensky, co-principal investigator. R.M.'s work was supported in part by Research Career Award KOI-MHOI754 and Schizophrenia Research Training Grant T32-MHI8870 from the National Institute of Mental Health, Rockville, MD, and by a National Alliance for Research on Schizophrenia and Depression Young Investigator Award. E.S.'s work was supported in part by Schizophrenia Research Center Grant 5P20-MH50727 and the Lieber Center. We thank Jack Gorman, MD, Evelyn Bromet, PhD, Alex Cohen, PhD, and Pamela Collins, MD for helpful comments on earlier versions of this paper.

\section{REFERENCES}

American Psychiatric Association (1994) Diagnostic and Statistical Manual of Mental Disorders (4th edition) (DSM-IV). Washington, DC: APA.

Collins, P. Y., Varma, V. K., Wig, N. N., et al (1999) Fever and acute brief psychosis in urban and rural settings in north India. British Journal of Psychiatry, 174, 520-524.

Day, R., Nielsen, J. A., Korten, A., et al (1987) Stressful life events preceding the acute onset of schizophrenia: A cross-national study from the World Health Organization. Culture, Medicine and Psychiatry, II 123-205.

Guinness, E. A. (1992) II. Brief reactive psychosis and the major functional psychoses: Descriptive case studies

\section{CLINICAL IMPLICATIONS}

- The modal duration of remitting psychoses with acute onset is 2-4 months.

- Mainly because of their duration, few of the cases met the criteria for ICD-I0 acute and transient psychotic disorders (ATPD).

- The ICD-I0 criteria for ATPD, especially in regard to duration, need to be refined so as to better capture the cases of remitting psychoses with acute onset.

\section{LIMITATIONS}

- The number of developing country sites among the incidence sites of the study was limited to two in north India.

- 'Acute onset' in this study was defined as onset within I week, whereas the ICD10 definition of acute onset for ATPD is onset within 2 weeks.

- Ratings of mode of onset and duration of illness were often based on information obtained from a key informant and these data could not always be verified by other means.

RAMIN MOJTABAI, MD, EZRA SUSSER, MD, Department of Psychiatry, Columbia University and New York State Psychiatric Institute; VIJOY VARMA, MD, Post-Graduate Institute of Medical Education and Research, Chandigarh, India

Correspondence: Ramin Mojtabai, MD, PhD, 200 Haven Avenue, Apt. 6P, New York, NY 10033, USA. Fax: (212) 78I-6345; e-mail:rm322@columbia.edu

(First received 29 July 1999, final revision 10 November 1999, accepted 10 November 1999)

in Africa. British Journal of Psychiatry, 160 (suppl. 16), 24 41.

Jablensky, A., Sartorius, N., Ernberg, G., et al (1992) Schizophrenia: Manifestations, incidence and course in different cultures. A World Health Organization Ten Country Study. Psychological Medicine: Monograph Supplement, 20. Cambridge: Cambridge University Press.

Jørgensen, P., Bennedsen, B., Christensen, J., et al (1997) Acute and transient psychotic disorder: A I-year follow-up study. Acta Psychiatrica Scandinavica, 96. I50-I54.

Leonhard, K. (1961) Cycloid psychoses - endogenous psychoses which are neither schizophrenic nor manicdepressive. Journal of Mental Science, 197, 633-648.

Malhotra, S., Varma, V. K., Misra, A. K., et al (1998) Onset of acute psychotic states in India: a study of sociodemographic, seasonal and biological factors. Acta Psychiatrica Scandinavica, 97, 125-131.

Pichot, P. (1986) The concept of "bouffée délirante" with special reference to the Scandinavian concept of reactive psychosis. Psychopathology, 19, 35-43.

Sartorius, N., Gulbinat, W., Harrison, G., et al (1996) Long-term follow-up of schizophrenia in 16 countries: A description of the International Study of Schizophrenia conducted by the World Health Organization. Social Psychiatry \& Psychiatric Epidemiology, 31, 249-258.

Susser, E. \& Wanderling, J. (1994) Epidemiology of nonaffective acute remitting psychosis vs schizophrenia: Sex and sociocultural setting. Archives of General Psychiatry, 5I, 294-30I.

\section{_ , Fenning, S., Jandorf, L., et al (1995a)}

Epidemiology, diagnosis, and course of brief psychoses. American Journal of Psychiatry, 152, 1743-1748.

\section{_ , Varma, V. K., Malhotra, S., et al (1995b)}

Delineation of acute and transient psychotic disorder in a developing country setting. British Journal of Psychiatry, 167, $216-219$

_ , _ , Mattoo, S. K., et al (1998) Long-term course of acute brief psychosis in a developing country setting. British Journal of Psychiatry, 173, 226-230.

Wing, J. K., Cooper, J. E. \& Sartorius, N. (1974) The Measurement and Classification of Psychiatric Symptoms. London: Cambridge University Press.

World Health Organization (1992) The ICD-10 Classification of Mental and Behavioral Disorders. Geneva: WHO. 